\section{Severe Vocal Cord Dysfunction: An Unusual Complication of Juvenile Dermatomyositis}

\section{To the Editor:}

Juvenile dermatomyositis (JDM) is a rare childhood disease characterized by varying degrees of skin and muscle inflammation. Other organ systems, including the gastrointestinal, pulmonary, cardiovascular, articular, ophthalmologic, and nervous systems, can be involved ${ }^{1}$. Pharyngeal, hypopharyngeal, and palatal muscles are frequently affected, leading to dysphagia and dysphonia ${ }^{1,2}$. To our knowledge, severe vocal cord dysfunction due to laryngeal muscle involvement has not been reported to date.

We describe a 15-year-old boy of Kurdish background who presented with a 6-month history of dryness, scaling, and fissuring to his palms and soles, which was initially diagnosed as palmoplantar keratoderma. Laboratory screening before he started oral retinoid therapy revealed elevated aspartate aminotransferase (AST) of $198 \mathrm{U} / 1$ (normal 10-45), alanine aminotransferase (ALT) $287 \mathrm{U} / 1$ (normal 10-40), and lactate dehydrogenase (LDH) 1133 U/1 (normal 360-730). Subsequent investigations showed antinuclear antibody titer of 1:640 for speckled pattern and 1:1280 nucleolar pattern, and the presence of anti PM-Scl antibodies. Over the subsequent 3 weeks, his rash progressed to involve the distal forearms and legs, and a new scaly erythematous rash appeared on the extensor surfaces of his fingers, elbows, and knees consistent with Gottron's papules. Over the next 2 weeks he developed progressive muscle weakness with inability to get out of bed, difficulty rising from a chair, dysphonia, dysphagia, and joint pain, and was referred for rheumatological assessment. Repeat studies revealed a normal complete blood count, an erythrocyte sedimentation rate of $43 \mathrm{~mm} / \mathrm{h}$, and further elevation of enzymes: AST 683 U/l, ALT 391 U/l, LDH 5349 U/l, with a creatine phosphokinase of 14,075 IU/1, and von Willebrand factor antigen level of 2.96 (normal 0.5-1.5). Magnetic resonance imaging (MRI) showed widespread signal abnormalities involving the obturators, psoas, rectus femoris, sartorius vastus lateralis and medialis, gluteus, and the adductor muscles (Figure 1). These findings were sufficient to establish the diagnosis of JDM without the need for muscle biopsy or electromyography. A swallowing study demonstrated both nasal regurgitation of barium and repeated significant aspiration; however, there was no anatomical abnormality or esophageal dysmotility (Figure 2).

Following an episode of aspiration, the patient deteriorated and required intubation for 13 days and then bi-level positive airway pressure

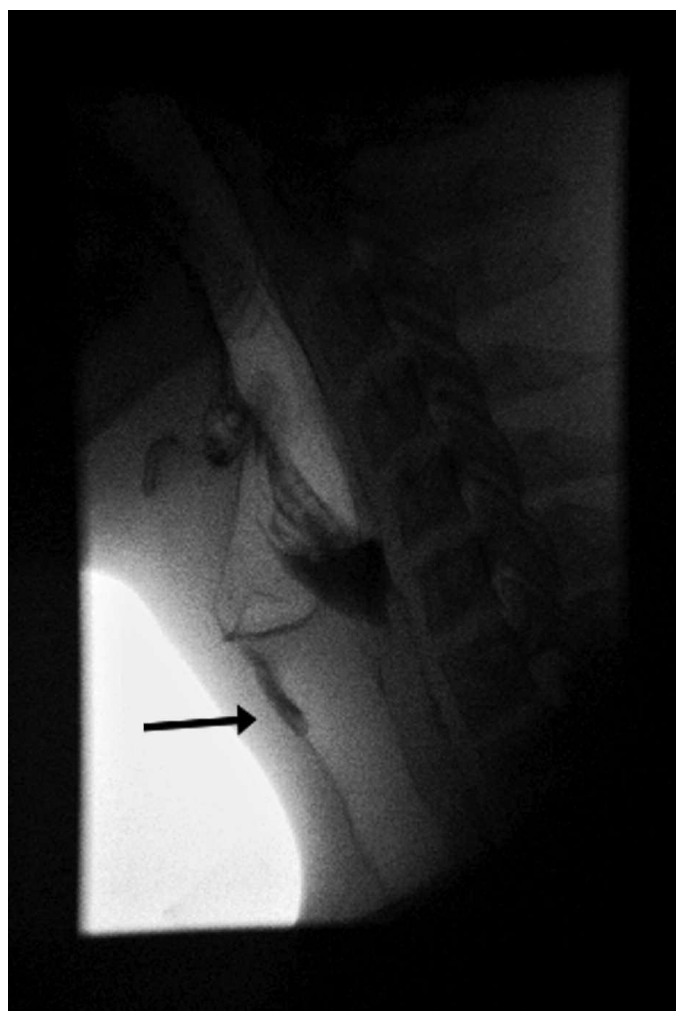

Figure 2. Swallowing study shows aspiration with barium in larynx below vocal cord level (arrow).

(BiPAP) for 6 months. The BiPAP was required only at night for hypoventilation due to respiratory muscle weakness. After extubation he could not vocalize and laryngoscopy identified lack of movement of both vocal cords in an abducted position and extensive loss of laryngeal musculature. $\mathrm{He}$ received aggressive treatment including intravenous methylprednisolone (1000 $\mathrm{mg}$ for 3 consecutive days), oral prednisone ( $2 \mathrm{mg} / \mathrm{kg} /$ day), weekly subcutaneous methotrexate $(25 \mathrm{mg})$, monthly intravenous cyclophosphamide $\left(500-1000 \mathrm{mg} / \mathrm{m}^{2} /\right.$ dose $\times 8$ doses), monthly intra-

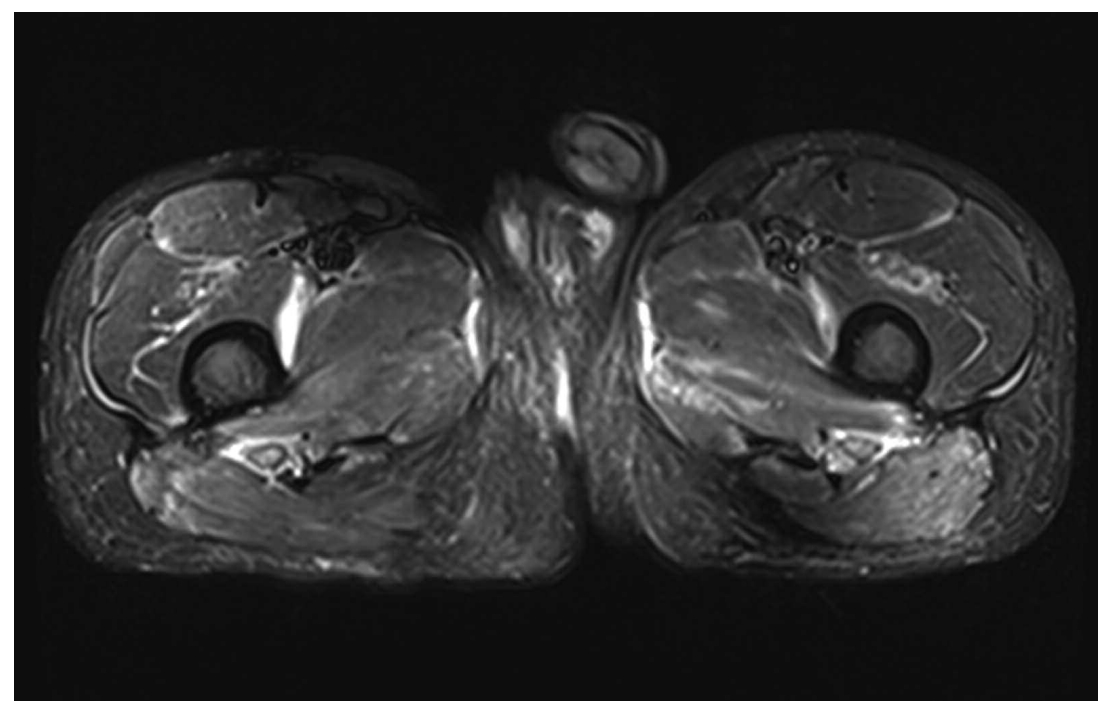

Figure 1. Magnetic resonance imaging short-tau inversion recovery scan shows increased signals in proximal thigh muscles. 
venous immunoglobulin ( $1 \mathrm{~g} / \mathrm{kg} /$ dose for $1 \mathrm{yr}$ ), and 3 courses of rituximab $\left(500 \mathrm{mg} / \mathrm{m}^{2}\right) 6$ months apart.

Serial laryngoscopy confirmed a slow recovery of right vocal cord motility after 3 months of treatment, coinciding with partial return of his voice. However, it took 14 months for complete recovery of motility of both vocal cords. Interestingly, the progressive recovery of his voice tracked with progressive global improvements assessed using the Childhood Myositis Assessment Scale, with scores increasing from 14 at presentation to 25,43 , and 49 at 4,6 , and 12 months, respectively.

Serial swallowing studies documented severe weakness of bulbar muscles and silent aspiration necessitating exclusive tube feeding (nasogastric, then gastrostomy) for 16 months. At 16 months there was no evidence of laryngeal penetration or tracheal spill; however there was slow clearance of contrast from the valleculae.

Vocal cord dysfunction may complicate intubation in $4.6 \%$ of cases; however, unlike in our patient, it is usually unilateral and transient, with a recovery time of 3 months ${ }^{3,4}$. An incorrectly positioned inflated endotracheal tube (ETT) cuff can result in compression of the anterior branch of the recurrent laryngeal nerve as it passes behind the thyroid cartilage to innervate the lateral cricoarytenoid muscle ${ }^{5}$. The evidence for intubation-induced laryngeal injury in our patient was low, and measures were undertaken to minimize this. Minimal occluding volume in a cuffed ETT size 7.5 was ensured at all times. ETT depth was kept at $24 \mathrm{~cm}$ at the mouth to ensure the cuff was in the trachea and not in the larynx. Moreover, there was no evidence of laryngeal trauma (edema, ulceration, laceration, or granuloma) on the initial laryngoscopy.

In nasogastric tube syndrome, the midline nasogastric tube may cause post-cricoid inflammation/ulceration and neuropraxia of the posterior division of recurrent laryngeal nerve, and subsequent vocal cord abduction paralysis ${ }^{5,6,7}$. Patients with this syndrome typically develop throat pain, stridor, and upper airway obstruction, and our patient had none of these features. Radiographs confirmed that the nasogastric tube was lateral to midline to prevent nasogastric tube syndrome.

Polyneuropathy in JDM is extremely rare, described only in case reports where there was never laryngeal involvement ${ }^{8,9,10}$. MRI of the brain and spine had ruled out a central neurological lesion that could lead to vocal cord paralysis.

We describe a patient with JDM and severe vocal cord dysfunction. We believe his vocal cord dysfunction was due to involvement of the laryngeal musculature and this represents a rare complication of this disease.
HOSAM O. ALTHAGAFI, MBChB, SSC-Ped, CABP, FRCPC; KIMBERLY MORISHITA, MD, MHSc, FRCPC; ROSS E. PETTY, CM, MD, PhD, FAAP, FRCPC, Division of Rheumatology, Department of Pediatrics, British Columbia's Children's Hospital and the University of British Columbia, 4480 Oak Street, Vancouver, British Columbia V6H 3V4, Canada. Address correspondence to Dr. H.O. Althagafi; E-mail: hosamthagafi@yahoo.com

\section{REFERENCES}

1. Ramanan AV, Feldman BM. Clinical features and outcomes of juvenile dermatomyositis and other childhood myositis syndromes. Rheum Dis Clin N Am 2002;28:833-57.

2. McCann LJ, Juggins AD, Maillard SM, Wedderburn LR, Davidson JE, Murray KJ, et al, on behalf of the Juvenile Dermatomyositis Research Group. The Juvenile Dermatomyositis National Registry and Repository (UK and Ireland) - Clinical characteristics of children recruited within the first $5 \mathrm{yr}$. Rheumatology 2006;45:1255-60.

3. Esteller-More E, Ibanez J, Matino E, Adema JM, Nolla M, Quer IM. Prognostic factors in laryngotracheal injury following intubation and/or tracheotomy in ICU patients. Eur Arch Oto-Rhino-Laryngol 2005;262:880-3.

4. Kikura M, Suzuki K, Itagaki T, Takada T, Sato S. Age and comorbidity as risk factors for vocal cord paralysis associated with tracheal intubation. Br J Anaesth 2007;98:524-30.

5. Friedman M, Toriumi DM. Esophageal stethoscope: Another possible cause of vocal cord paralysis. Arch Otolaryngol Head Neck Surg 1989;115:95-8.

6. Sofferman RA, Haisch CE, Kirchner JA, Hardin NJ. The nasogastric tube syndrome. Laryngoscope 1990;100:962-8.

7. Isozaki E, Tobisawa S, Naito R, Mizutani T, Hayashi H. A variant form of nasogastric tube syndrome. Intern Med 2005;44:1286-90.

8. Vogelgesang SA, Gutierrez J, Klipple GL, Katona IM. Polyneuropathy in juvenile dermatomyositis. J Rheumatol 1995;22:1369-72.

9. Laraki R, Bletry O, Godeau P. Polymyositis/dermatomyositis and polyneuropathy, or, does neuromyositis exist? J Rheumatol 1996;23:2186-7.

10. Nomura M, Watanabe T, Mikami H, Ishikawa H, Yasui K, Yamazaki T, et al. Adult dermatomyositis with severe polyneuropathy: Does neuromyositis exist? Neurol Sci 2010;31:373-6.

J Rheumatol 2013;40:5; doi:10.3899/jrheum.121338 